\title{
Direct Search for Charged Higgs Bosons in Decays of Top Quarks
}

V. M. Abazov, ${ }^{23}$ B. Abbott,${ }^{58}$ A. Abdesselam,,${ }^{11}$ M. Abolins,${ }^{51}$ V. Abramov, ${ }^{26}$ B. S. Acharya, ${ }^{17}$ D. L. Adams, ${ }^{60}$ M. Adams, ${ }^{38}$ S. N. Ahmed,${ }^{21}$ G. D. Alexeev, ${ }^{23}$ G. A. Alves,${ }^{2}$ N. Amos,${ }^{50}$ E. W. Anderson, ${ }^{43}$ M. M. Baarmand, ${ }^{55}$ V. V. Babintsev, ${ }^{26}$ L. Babukhadia,${ }^{55}$ T. C. Bacon, ${ }^{28}$ A. Baden, ${ }^{47}$ B. Baldin, ${ }^{37}$ P.W. Balm,${ }^{20}$ S. Banerjee,,${ }^{17}$ E. Barberis, ${ }^{30}$ P. Baringer ${ }^{44}$ J. Barreto, ${ }^{2}$ J. F. Bartlett, ${ }^{37}$ U. Bassler,${ }^{12}$ D. Bauer, ${ }^{28}$ A. Bean,${ }^{44}$ M. Begel, ${ }^{54}$ A. Belyaev, ${ }^{25}$ S. B. Beri, ${ }^{15}$ G. Bernardi, ${ }^{12}$ I. Bertram, ${ }^{27}$ A. Besson, ${ }^{9}$ R. Beuselinck, ${ }^{28}$ V. A. Bezzubov, ${ }^{26}$ P. C. Bhat, ${ }^{37}$ V. Bhatnagar, ${ }^{11}$ M. Bhattacharjee, ${ }^{55}$ G. Blazey, ${ }^{39}$ S. Blessing, ${ }^{35}$ A. Boehnlein, ${ }^{37}$ N. I. Bojko, ${ }^{26}$ F. Borcherding, ${ }^{37}$ K. Bos, ${ }^{20}$ A. Brandt,${ }^{60}$

R. Breedon, ${ }^{31}$ G. Briskin, ${ }^{59}$ R. Brock,${ }^{51}$ G. Brooijmans, ${ }^{37}$ A. Bross,${ }^{37}$ D. Buchholz,${ }^{40}$ M. Buehler ${ }^{38}$ V. Buescher, ${ }^{14}$

V. S. Burtovoi, ${ }^{26}$ J. M. Butler, ${ }^{48}$ F. Canelli, ${ }^{54}$ W. Carvalho, ${ }^{3}$ D. Casey,${ }^{51}$ Z. Casilum, ${ }^{55}$ H. Castilla-Valdez, ${ }^{19}$

D. Chakraborty, ${ }^{55}$ K. M. Chan, ${ }^{54}$ S. V. Chekulaev, ${ }^{26}$ D. K. Cho, ${ }^{54}$ S. Choi, ${ }^{34}$ S. Chopra, ${ }^{56}$ J. H. Christenson, ${ }^{37}$ M. Chung, ${ }^{38}$ D. Claes, ${ }^{52}$ A. R. Clark, ${ }^{30}$ J. Cochran,,${ }^{34}$ L. Coney, ${ }^{42}$ B. Connolly, ${ }^{35}$ W. E. Cooper, ${ }^{37}$ D. Coppage, ${ }^{44}$ M. A. C. Cummings, ${ }^{3,9}$ D. Cutts, ${ }^{59}$ G. A. Davis,${ }^{54}$ K. Davis,${ }^{29}$ K. De ${ }^{60}$ S. J. de Jong, ${ }^{21}$ K. Del Signore, ${ }^{50}$ M. Demarteau, ${ }^{37}$ R. Demina, ${ }^{45}$ P. Demine, ${ }^{9}$ D. Denisov,${ }^{37}$ S. P. Denisov, ${ }^{26}$ S. Desai,,${ }^{55}$ H. T. Diehl,${ }^{37}$ M. Diesburg,,${ }^{37}$ G. Di Loreto, ${ }^{51}$ S. Doulas, ${ }^{49}$ P. Draper,${ }^{60}$ Y. Ducros,${ }^{13}$ L. V. Dudko, ${ }^{25}$ S. Duensing, ${ }^{21}$ L. Duflot, ${ }^{11}$ S. R. Dugad, ${ }^{17}$ A. Dyshkant, ${ }^{26}$ D. Edmunds, ${ }^{51}$ J. Ellison, ${ }^{34}$ V. D. Elvira, ${ }^{37}$ R. Engelmann, ${ }^{55}$ S. Eno, ${ }^{47}$ G. Eppley, ${ }^{62}$ P. Ermolov, ${ }^{25}$ O. V. Eroshin, ${ }^{26}$ J. Estrada, ${ }^{54}$ H. Evans, ${ }^{53}$ V. N. Evdokimov, ${ }^{26}$ T. Fahland, ${ }^{33}$ S. Feher, ${ }^{37}$ D. Fein, ${ }^{29}$ T. Ferbel, ${ }^{54}$ F. Filthaut, ${ }^{21}$ H. E. Fisk, ${ }^{37}$ Y. Fisyak, ${ }^{56}$ E. Flattum, ${ }^{37}$ F. Fleuret, ${ }^{30}$ M. Fortner, ${ }^{39}$ K. C. Frame, ${ }^{51}$ S. Fuess,${ }^{37}$ E. Gallas, ${ }^{37}$ A. N. Galyaev, ${ }^{26}$ M. Gao, ${ }^{53}$ V. Gavrilov, ${ }^{24}$ R. J. Genik II, ${ }^{27}$ K. Genser, ${ }^{37}$ C. E. Gerber, ${ }^{38}$ Y. Gershtein, ${ }^{59}$ R. Gilmartin, ${ }^{35}$ G. Ginther ${ }^{54}$ B. Gómez, ${ }^{5}$ G. Gómez,${ }^{47}$ P. I. Goncharov, ${ }^{26}$ J. L. González Solís, ${ }^{19}$ H. Gordon, ${ }^{56}$ L. T. Goss, ${ }^{61}$ K. Gounder, ${ }^{37}$ A. Goussiou, ${ }^{55}$ N. Graf, ${ }^{56}$ G. Graham, ${ }^{47}$ P. D. Grannis, ${ }^{55}$ J. A. Green, ${ }^{43}$ H. Greenlee, ${ }^{37}$ S. Grinstein, ${ }^{1}$ L. Groer ${ }^{53}$ S. Grünendahl, ${ }^{37}$ A. Gupta, ${ }^{17}$ S. N. Gurzhiev, ${ }^{26}$ G. Gutierrez ${ }^{37}$ P. Gutierrez, ${ }^{58}$ N. J. Hadley, ${ }^{47}$ H. Haggerty, ${ }^{37}$ S. Hagopian, ${ }^{35}$ V. Hagopian, ${ }^{35}$ R. E. Hall ${ }^{32}$ P. Hanlet, ${ }^{49}$ S. Hansen, ${ }^{37}$ J. M. Hauptman, ${ }^{43}$ C. Hays,${ }^{53}$ C. Hebert ${ }^{44}$ D. Hedin, ${ }^{39}$ A. P. Heinson, ${ }^{34}$ U. Heintz,${ }^{48}$ T. Heuring,${ }^{35}$ M. D. Hildreth ${ }^{42}$ R. Hirosky, ${ }^{63}$ J. D. Hobbs,${ }^{55}$ B. Hoeneisen, ${ }^{8}$ Y. Huang, ${ }^{50}$ R. Illingworth, ${ }^{28}$ A. S. Ito, ${ }^{37}$ M. Jaffré, ${ }^{11}$ S. Jain, ${ }^{17}$ R. Jesik, ${ }^{41}$ K. Johns, ${ }^{29}$ M. Johnson, ${ }^{37}$ A. Jonckheere, ${ }^{37}$ M. Jones,${ }^{36}$ H. Jöstlein, ${ }^{37}$ A. Juste, ${ }^{37}$ S. Kahn, ${ }^{56}$ E. Kajfasz, ${ }^{10}$ A. M. Kalinin, ${ }^{23}$ D. Karmanov, ${ }^{25}$ D. Karmgard, ${ }^{42}$ R. Kehoe, ${ }^{51}$ A. Kharchilava, ${ }^{42}$ S. K. Kim, ${ }^{18}$ B. Klima, ${ }^{37}$ B. Knuteson, ${ }^{30}$ W. Ko, ${ }^{31}$ J. M. Kohli, ${ }^{15}$ A. V. Kostritskiy,${ }^{26}$ J. Kotcher, ${ }^{56}$ A. V. Kotwal, ${ }^{53}$ A. V. Kozelov, ${ }^{26}$ E. A. Kozlovsky, ${ }^{26}$ J. Krane, ${ }^{43}$ M. R. Krishnaswamy, ${ }^{17}$ P. Krivkova, ${ }^{6}$

S. Krzywdzinski, ${ }^{37}$ M. Kubantsev, ${ }^{45}$ S. Kuleshov, ${ }^{24}$ Y. Kulik, ${ }^{55}$ S. Kunori,${ }^{47}$ A. Kupco, ${ }^{7}$ V. E. Kuznetsov, ${ }^{34}$

G. Landsberg, ${ }^{59}$ A. Leflat, ${ }^{25}$ C. Leggett, ${ }^{30}$ F. Lehner, ${ }^{37}$ J. Li,${ }^{60}$ Q. Z. Li,${ }^{37}$ J. G. R. Lima, ${ }^{3}$ D. Lincoln, ${ }^{37}$ S. L. Linn, ${ }^{35}$ J. Linnemann, ${ }^{51}$ R. Lipton, ${ }^{37}$ A. Lucotte, ${ }^{9}$ L. Lueking, ${ }^{37}$ C. Lundstedt,${ }^{52}$ C. Luo, ${ }^{41}$ A. K. A. Maciel,,${ }^{3,9}$ R. J. Madaras, ${ }^{30}$ V. L. Malyshev, ${ }^{23}$ V. Manankov, ${ }^{25}$ H. S. Mao, ${ }^{4}$ T. Marshall, ${ }^{41}$ M. I. Martin, ${ }^{37}$ R. D. Martin, ${ }^{38}$ K. M. Mauritz, ${ }^{43}$ B. May, ${ }^{40}$ A. A. Mayorov, ${ }^{41}$ R. McCarthy, ${ }^{55}$ J. McDonald ${ }^{35}$ T. McMahon, ${ }^{57}$ H. L. Melanson, ${ }^{37}$ M. Merkin, ${ }^{25}$ K. W. Merritt, ${ }^{37}$

C. Miao, ${ }^{59}$ H. Miettinen, ${ }^{62}$ D. Mihalcea, ${ }^{58}$ C. S. Mishra, ${ }^{37}$ N. Mokhov, ${ }^{37}$ N. K. Mondal, ${ }^{17}$ H.E. Montgomery, ${ }^{37}$

R. W. Moore,${ }^{51}$ M. Mostafa, ${ }^{1}$ H. da Motta, ${ }^{2}$ E. Nagy, ${ }^{10}$ F. Nang, ${ }^{29}$ M. Narain, ${ }^{48}$ V. S. Narasimham, ${ }^{17}$ H. A. Neal, ${ }^{50}$ J. P. Negret, ${ }^{5}$ S. Negroni, ${ }^{10}$ T. Nunnemann, ${ }^{37}$ D. O'Neil,${ }^{51}$ V. Oguri, ${ }^{3}$ B. Olivier, ${ }^{12}$ N. Oshima, ${ }^{37}$ P. Padley, ${ }^{62}$ L. J. Pan, ${ }^{40}$

K. Papageorgiou, ${ }^{28}$ A. Para,${ }^{37}$ N. Parashar, ${ }^{49}$ R. Partridge,${ }^{59}$ N. Parua, ${ }^{55}$ M. Paterno, ${ }^{54}$ A. Patwa,${ }^{55}$ B. Pawlik,${ }^{22}$ J. Perkins,${ }^{60}$ M. Peters,${ }^{36}$ O. Peters, ${ }^{20}$ P. Pétroff,,${ }^{11}$ R. Piegaia, ${ }^{1}$ H. Piekarz,${ }^{35}$ B. G. Pope,${ }^{51}$ E. Popkov,${ }^{48}$ H. B. Prosper ${ }^{35}$ S. Protopopescu, ${ }^{56}$ J. Qian, ${ }^{50}$ R. Raja, ${ }^{37}$ S. Rajagopalan, ${ }^{56}$ E. Ramberg,${ }^{37}$ P. A. Rapidis, ${ }^{37}$ N. W. Reay, ${ }^{45}$ S. Reucroft, ${ }^{49}$ J. Rha, ${ }^{34}$ M. Ridel, ${ }^{11}$ M. Rijssenbeek,${ }^{55}$ T. Rockwell,${ }^{51}$ M. Roco,${ }^{37}$ P. Rubinov,${ }^{37}$ R. Ruchti,${ }^{42}$ J. Rutherfoord, ${ }^{29}$

B. M. Sabirov, ${ }^{23}$ A. Santoro, ${ }^{2}$ L. Sawyer,${ }^{46}$ R. D. Schamberger, ${ }^{55}$ H. Schellman, ${ }^{40}$ A. Schwartzman, ${ }^{1}$ N. Sen, ${ }^{62}$

E. Shabalina, ${ }^{25}$ R. K. Shivpuri, ${ }^{16}$ D. Shpakov, ${ }^{49}$ M. Shupe, ${ }^{29}$ R. A. Sidwell, ${ }^{45}$ V. Simak, ${ }^{7}$ H. Singh, ${ }^{34}$ J. B. Singh, ${ }^{15}$ V. Sirotenko, ${ }^{37}$ P. Slattery, ${ }^{54}$ E. Smith, ${ }^{58}$ R. P. Smith, ${ }^{37}$ R. Snihur, ${ }^{40}$ G. R. Snow, ${ }^{52}$ J. Snow, ${ }^{57}$ S. Snyder, ${ }^{56}$ J. Solomon, ${ }^{38}$ V. Sorín, ${ }^{1}$ M. Sosebee, ${ }^{60}$ N. Sotnikova, ${ }^{25}$ K. Soustruznik, ${ }^{6}$ M. Souza, ${ }^{2}$ N. R. Stanton, ${ }^{45}$ G. Steinbrück,${ }^{53}$

R. W. Stephens, ${ }^{60}$ F. Stichelbaut, ${ }^{56}$ D. Stoker ${ }^{33}$ V. Stolin, ${ }^{24}$ D. A. Stoyanova, ${ }^{26}$ M. Strauss, ${ }^{58}$ M. Strovink, ${ }^{30}$ L. Stutte, ${ }^{37}$

A. Sznajder, ${ }^{3}$ W. Taylor, ${ }^{55}$ S. Tentindo-Repond, ${ }^{35}$ S. M. Tripathi, ${ }^{31}$ T. G. Trippe, ${ }^{30}$ A. S. Turcot, ${ }^{56}$ P. M. Tuts, ${ }^{53}$

P. van Gemmeren, ${ }^{37}$ V. Vaniev, ${ }^{26}$ R. Van Kooten, ${ }^{41}$ N. Varelas, ${ }^{38}$ L. S. Vertogradov, ${ }^{23}$ A. A. Volkov, ${ }^{26}$ A. P. Vorobiev, ${ }^{26}$ H. D. Wahl, ${ }^{35}$ H. Wang, ${ }^{40}$ Z.-M. Wang, ${ }^{55}$ J. Warchol, ${ }^{42}$ G. Watts, ${ }^{64}$ M. Wayne, ${ }^{42}$ H. Weerts, ${ }^{51}$ A. White,${ }^{60}$ J. T. White,${ }^{61}$ D. Whiteson, ${ }^{30}$ J. A. Wightman, ${ }^{43}$ D. A. Wijngaarden, ${ }^{21}$ S. Willis, ${ }^{39}$ S. J. Wimpenny, ${ }^{34}$ J. Womersley,${ }^{37}$ D. R. Wood, ${ }^{49}$

R. Yamada ${ }^{37}$ P. Yamin,${ }^{56}$ T. Yasuda,${ }^{37}$ Y. A. Yatsunenko, ${ }^{23}$ K. Yip, ${ }^{56}$ S. Youssef,,${ }^{35}$ J. Yu,${ }^{37}$ Z. Yu, ${ }^{40}$ M. Zanabria, ${ }^{5}$ H. Zheng, ${ }^{42}$ Z. Zhou, ${ }^{43}$ M. Zielinski, ${ }^{54}$ D. Zieminska, ${ }^{41}$ A. Zieminski, ${ }^{41}$ V. Zutshi, ${ }^{54}$ E. G. Zverev, ${ }^{25}$ and A. Zylberstejn ${ }^{13}$ 


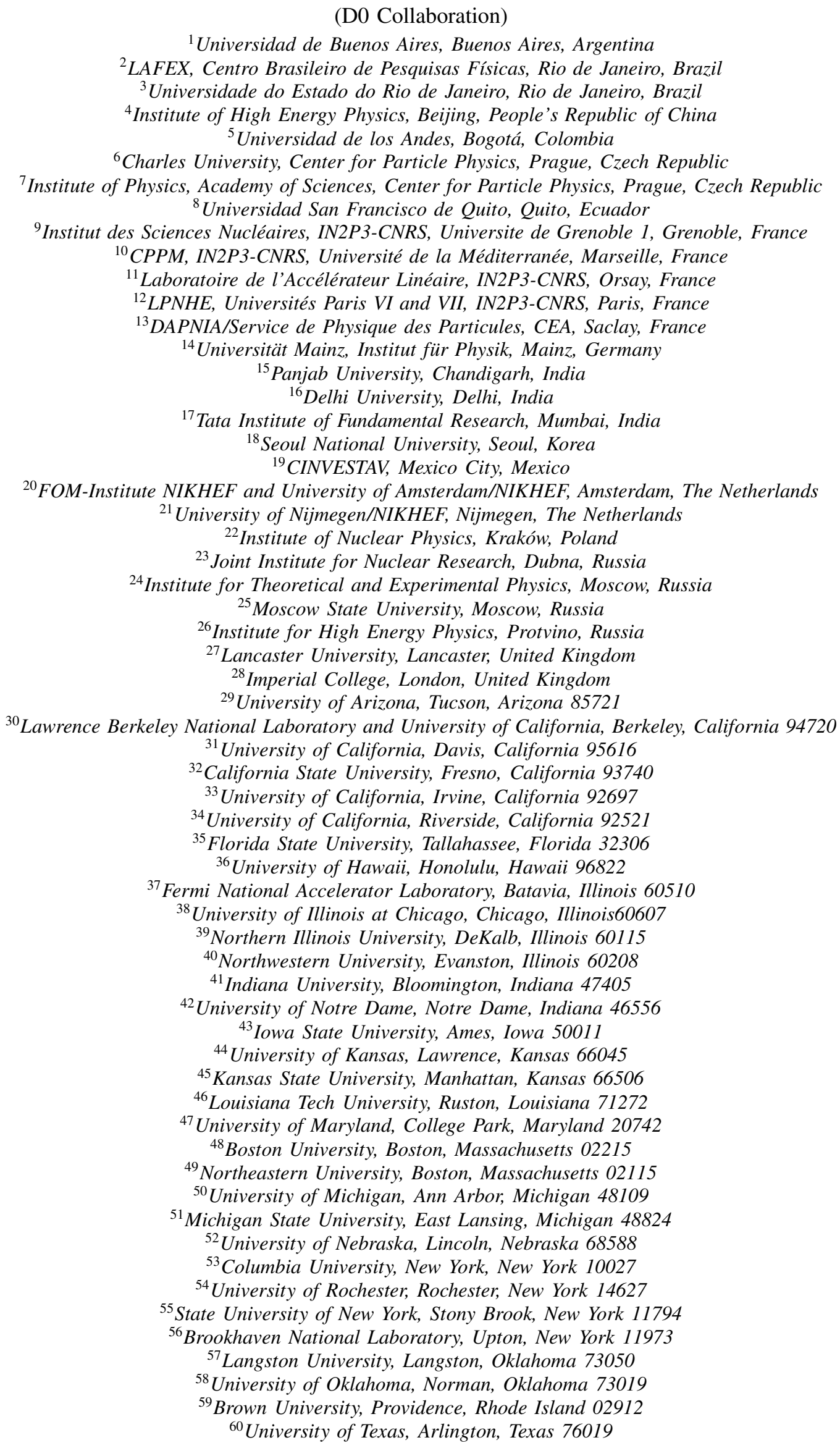


${ }^{61}$ Texas A\&M University, College Station, Texas 77843
${ }^{62}$ Rice University, Houston, Texas 77005
${ }^{63}$ University of Virginia, Charlottesville, Virginia 22901
${ }^{64}$ University of Washington, Seattle, Washington 98195
(Received 20 February 2001; published 29 March 2002)

We present a search for charged Higgs bosons in decays of pair-produced top quarks in $p \bar{p}$ collisions at $\sqrt{s}=1.8 \mathrm{TeV}$ recorded by the D0 detector at the Fermilab Tevatron collider. With no evidence for signal, we exclude most regions of the $\left(M_{H^{ \pm}}, \tan \beta\right)$ parameter space where the decay $t \rightarrow \mathrm{H}^{+} b$ has a branching fraction $>0.36$ and $B\left(H^{ \pm} \rightarrow \tau \nu_{\tau}\right)$ is large.

The standard model (SM) relies on the Higgs mechanism for gauge-invariant generation of particle masses [1]. It contains a single complex scalar doublet field, whose only observable particle is the neutral Higgs boson $H^{0}$. At present, no data limit the Higgs sector to a single doublet. In this Letter, we examine predictions of a two-Higgsdoublet model (THDM) that couples one doublet to uptype quarks and neutrinos, and the other to down-type quarks and charged leptons (type-II model), just as in the minimal supersymmetric extension of the SM [2]. For such coupling, flavor changing neutral currents are absent at tree level [2]. The additional degrees of freedom in this model provide a total of five observable Higgs fields: two neutral CP-even scalars $h^{0}$ and $H^{0}$, a neutral CP-odd scalar $A^{0}$, and two charged scalars $H^{ \pm}$. In what follows, we report on a search for evidence of an extension of the Higgs sector, in the form of a $H^{ \pm}$boson, with the relevant parameters being its mass, $M_{H^{ \pm}}$, and the ratio of the vacuum expectation values of the doublets, $\tan \beta$.

In the SM, the primary decay of the $t$ quark is $t \rightarrow W^{+} b$. The addition of the second Higgs doublet provides the $t \rightarrow$ $H^{+} b$ mode, with a branching fraction $B\left(t \rightarrow H^{+} b\right) \propto$ $\left(m_{t}^{2} \cot ^{2} \beta+m_{b}^{2} \tan ^{2} \beta\right)+4 m_{t}^{2} m_{b}^{2}$. This function has a minimum when $\tan \beta=\sqrt{m_{t} / m_{b}}$, and is symmetric in $\log _{10}(\tan \beta)$ about this point. If $\tan \beta$ differs by about an order of magnitude from $\sqrt{m_{t} / m_{b}}$, the branching fraction becomes large, and decreases as $M_{H^{ \pm}}$increases. In this analysis, we assume $B\left(t \rightarrow W^{+} b\right)+B\left(t \rightarrow H^{+} b\right)=1$. The masses of the three neutral scalars are assumed to be large enough to be suppressed in $H^{ \pm}$decays. Also, at tree level, there are no direct $H^{ \pm}$couplings to SM vector bosons. The only available decays of $H^{ \pm}$are therefore fermionic, with coupling proportional to fermion mass. For $M_{H^{ \pm}}$below $\approx 110 \mathrm{GeV}, B\left(H^{+} \rightarrow \tau^{+} \nu\right) \approx 0.96$ for $\tan \beta>2$, and $B\left(H^{+} \rightarrow c \bar{s}\right) \approx 1$ for $\tan \beta<0.4$. Because of large coupling to the top quark [3], $B\left(H^{+} \rightarrow\right.$ $\left.t^{*} \bar{b} \rightarrow W^{+} b \bar{b}\right)$ becomes important and eventually dominant at higher values of $M_{H^{ \pm}}$for $\tan \beta<\sqrt{m_{t} / m_{b}}$.

D0 has carried out two searches for $t \rightarrow H^{+} b$. An indirect search, which has been published [4], looked for a decrease in the $t \bar{t} \rightarrow W^{+} W^{-} b \bar{b}$ signal expected from the $\mathrm{SM}$, and the direct search, reported here, that searches for the $H^{ \pm} \rightarrow \tau^{ \pm} \nu$ decay mode. Direct searches have been carried out by CERN $e^{+} e^{-}$-collider LEP experiments, and report a combined lower limit on $M_{H^{ \pm}}$of $78.6 \mathrm{GeV}$ [5]. CDF has also reported a direct search for $H^{ \pm}$, setting an upper limit on $B\left(t \rightarrow H^{+} b\right)$ in the range of 0.5 to 0.6 at 95\% confidence level (C.L.) for masses in the range 60 to $160 \mathrm{GeV}$, assuming $B\left(H^{+} \rightarrow \tau \nu_{\tau}\right)=1$ [6].

In addition to the limits from the Tevatron and LEP, limits have also been published based on quantum corrections for type-II THDM in other processes. CLEO sets a limit of $M_{H^{ \pm}}>\left[244+63 /(\tan \beta)^{1 / 3}\right] \mathrm{GeV}$ at the $95 \%$ C.L. from their inclusive measurement of $b \rightarrow s \gamma$ [7]. The L3 limit [8] on $B \rightarrow \tau+\nu_{\tau}$, leads Ref. [9] to set a 90\% C.L. limit of $0.27 \mathrm{GeV}^{-1}>(\tan \beta) / M_{H^{ \pm}}$. Finally, the branching ratios of $\tau \rightarrow \nu_{\tau} K$ and $K \rightarrow \nu_{\ell}(\gamma)$, yields a limit of $0.21 \mathrm{GeV}^{-1}>(\tan \beta) / M_{H^{ \pm}}$at the $90 \%$ C.L. [10]. Although these limits exclude a larger part of available parameter space than our study, because of the difficulty of the measurements and ambiguities in theory, it is important to search for objects such as the $H^{ \pm}$in all possible channels, and not to defer entirely to theory.

This analysis uses the same formulation and Monte Carlo (MC) tools as our indirect search. The theory is a leading-order perturbative calculation, requiring the $t \rightarrow$ $H^{+} b$ coupling to be $<1$, which limits the validity of our search to $0.3<\tan \beta<150$. In addition, the calculation is unreliable for small $\left|m_{t}-M_{H^{ \pm}}\right|$and for large decay widths for $t$ and $H^{ \pm}$. This further limits our search to $M_{H^{ \pm}}<160 \mathrm{GeV}$ and $B\left(t \rightarrow H^{+} b\right)<0.9$.

A direct search for $H^{ \pm}$divides naturally into two regions [11]: (1) small $\tan \beta$, where final states are dominated by jets, with imbalance in transverse momentum $\left(E_{T}\right)$, and (2) large $\tan \beta$, where the final state contains up to two $\tau$ leptons and large missing transverse energy $\left(\mathbb{E}_{T}\right)$. Because at $\operatorname{small} \tan \beta$ there is background from multijet production, we concentrate on large $\tan \beta$ and $t \bar{t} \rightarrow \tau \bar{\tau} \nu_{\tau} \bar{\nu}_{\tau}+$ jets final states. The experimental signature for $t \rightarrow H^{+} b$ is nearly identical to that for $t \rightarrow W^{+} b$. We therefore rely on the expected increase in absolute yield of $\tau$ leptons at high $\tan \beta$ to differentiate between the two modes.

The $t \bar{t}$ data for this analysis were obtained from $p \bar{p}$ collisions at $\sqrt{s}=1.8 \mathrm{TeV}$ [12], and we consider both $t \bar{t} \rightarrow H^{+} H^{-} b \bar{b}$ and $t \bar{t} \rightarrow H^{ \pm} W^{\mp} b \bar{b}$ channels. Identification of the $\tau$ relies on its hadronic decay modes, consisting primarily of one or three charged hadrons in a narrow jet, often accompanied by photons from $\pi^{0}$ decays, and a $\nu_{\tau}$. There are two $b$ jets per event, and, when one of the top quarks decays to $W b$, there are also two light quark jets (we consider only hadronic $W$ modes). The event signature is therefore jets $+\mathbb{E}_{T}$, with a roughly spherical 


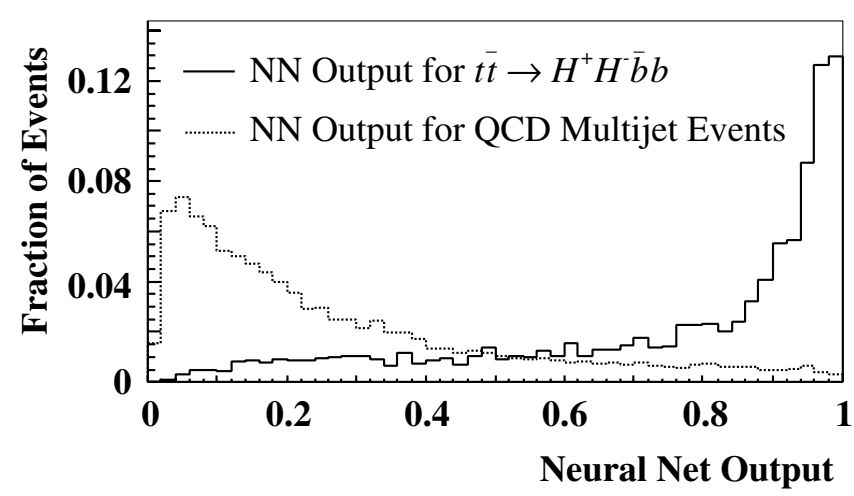

FIG. 1. NN output for $t \bar{t} \rightarrow H^{+} H^{-} b \bar{b}$ MC signal and multijet background, normalized to the same area.

distribution in the detector, and at least one narrow jet. Consequently, we rely on a multijet $+\mathbb{E}_{T}$ trigger, which comprises $62.2 \pm 3.1 \mathrm{pb}^{-1}$ of integrated luminosity $(\mathcal{L})$. To reduce background, we use a set of loose selections, and then a neural network $(\mathrm{NN})$ for more restrictive cuts. The loose criteria require that the event have $\mathbb{E}_{T}>25 \mathrm{GeV}$, at least 4 jets, each with $E_{T}>20 \mathrm{GeV}$, but no more than 8 jets with $E_{T}>8 \mathrm{GeV}$.

We use a feed-forward NN [13] based on JETNET [14], with 3 input nodes, 7 hidden nodes, and 1 output node. The input variables are $\mathbb{E}_{T}$, and two of the three eigenvalues of the normalized momentum tensor. The $\mathrm{NN}$ is trained on both signal $\left(t \rightarrow H^{+} b\right)$, and background. The sample for training on signal, $t \bar{t} \rightarrow H^{+} H^{-} b \bar{b}$, is generated using ISAJET [15], with both $H^{+}$and $H^{-}$decaying to $\tau \nu_{\tau}$, and the $\tau$ leptons to hadrons and $\nu_{\tau}$. The response of the $\mathrm{NN}$ is relatively insensitive to the $M_{H^{ \pm}}$, we therefore use only one value, $M_{H^{ \pm}}=95 \mathrm{GeV}$. The same $\mathrm{NN}$ is also used for classifying $t \bar{t} \rightarrow H^{ \pm} W^{\mp} b \bar{b}$ channels, since the efficiency for this channel is comparable to that of the training sample.

The primary sources of background are mismeasured multijet events, and $W+\geq 3$ jet events. We therefore train the NN on a sample of 25000 multijet events from data; even if the $H^{ \pm}$exists, $\approx 1$ event is added to the sample. The $W+$ jets background is modeled using VECBOS [16] for parton production, and ISAJET for hadronization. Figure 1 shows the separation achieved for $H^{ \pm}$signal relative to our main background from multijet events. The chosen $\mathrm{NN}$ cutoff of 0.91 is based on a series of MC experiments used to determine the maximum sensitivity for $H^{ \pm}$. In the absence of signal, this also provides the maximum excluded area in $\left(M_{H^{ \pm}}, \tan \beta\right)$ space.

After applying the NN selection, we require that events have at least one hadronically decaying $\tau$ lepton. The selection used in this analysis follows that of our $W \rightarrow \tau \nu_{\tau}$ study [17]. The principal requirement being the identification of one narrow jet in each event $\left(\sqrt{\sigma_{\eta}^{2}+\sigma_{\phi}^{2}} \leq\right.$ 0.25 , where the $\sigma$ correspond to the jet widths in $\eta$, pseudorapidity, and $\phi$, azimuthal angle), with 1 to 7 charged tracks, $10<E_{T}<60 \mathrm{GeV}$ for jets of cone $R=$ $\sqrt{\Delta \eta^{2}+\Delta \phi^{2}}=0.5$, and rejection of events with elec-
TABLE I. Cumulative efficiencies (\%) after the three stages of event selection for $H^{ \pm}$signal and background, for $M_{H^{ \pm}}=$ $95 \mathrm{GeV}$. The errors are statistical and systematic uncertainties added in quadrature. Event types are (1) $t \bar{t} \rightarrow W^{ \pm} H^{\overline{ }} b \bar{b}$, $W \rightarrow q \bar{q}^{\prime}, H \rightarrow \tau \nu_{\tau}$; (2) $t \bar{t} \rightarrow H^{ \pm} H^{\mp} b \bar{b}, H \rightarrow \tau \nu_{\tau}$; (3) $t \bar{t} \rightarrow$ $W^{ \pm} W^{+} b \bar{b}, W \rightarrow \tau \nu_{\tau}, W \rightarrow q \bar{q}^{\prime}$; and (4) $W+\geq 3$ jets, $W \rightarrow$ $\tau \nu_{\tau}$, where we consider only $\tau \rightarrow$ jet decays.

\begin{tabular}{cccc}
\hline \hline Type & Loose selection & $\mathrm{NN}>0.91$ & $\tau$-id \\
\hline 1 & $50.0 \pm 1.7$ & $18.3 \pm 0.9$ & $5.0 \pm 1.0$ \\
2 & $35.2 \pm 1.6$ & $12.9 \pm 0.9$ & $5.5 \pm 1.0$ \\
3 & $45.1 \pm 2.0$ & $15.7 \pm 1.0$ & $3.8 \pm 0.8$ \\
4 & $0.65 \pm 0.04$ & $0.17 \pm 0.02$ & $0.04 \pm 0.01$ \\
\hline \hline
\end{tabular}

trons or muons Ref. [18]. In addition to the criteria in Ref. [17], we require that the discriminant $\chi_{b}^{2}-\chi_{s}^{2}>0$, where $\chi_{s}^{2}$ and $\chi_{b}^{2}$ are the $\chi^{2}$ determined from a covariance matrix calculated from $W \rightarrow \tau \nu_{\tau} \mathrm{MC}$, and a sample of multijet events, respectively. The $\chi^{2}$ for the multijet sample uses the leading jet in each event $\left(E_{T}>20 \mathrm{GeV}\right)$. To define the covariance matrix, we use the fact that $\tau$ jets are narrower than normal hadronic jets in the energy range of our search. The variables used are the energy in each of the first five layers of our calorimeter, the log of the total energy, the ratio of the sum of the transverse energy of the two calorimeter towers with highest $E_{T}$ to the total jet energy, and ratios of jet energies in the central $3 \times 3$ and $5 \times 5$ calorimeter towers to the total jet energy.

Because the measured values of $\sigma_{t \bar{t}}$ and $m_{t}$ are based on the assumption that $B\left(t \rightarrow W^{+} b\right)=1$, it may be regarded as specious to use either in calculating the expected number of events. For $t \bar{t}$ production, we therefore use a QCD calculation giving $\sigma_{t \bar{t}}=5.5 \mathrm{pb}$ [19-21]. Any possible contamination from $t \bar{t} \rightarrow H^{ \pm} W^{\mp} b \bar{b}$, would affect the D0 $m_{t}$ measurement by $<5 \%$ for $M_{H^{ \pm}}<140 \mathrm{GeV}$; we therefore use the value $m_{t}=175 \mathrm{GeV}[22,23]$. The selection efficiencies for signal and background are listed in Table I. Using this information, we expect $1.1 \pm 0.3$ events from $t \bar{t}, 0.9 \pm 0.3$ from $W+$ jets, and $3.2 \pm 1.5$ from mulitjet background, while we observe 3 events in the data. The jet energy, modeling of signal, and $\tau$ identification are the

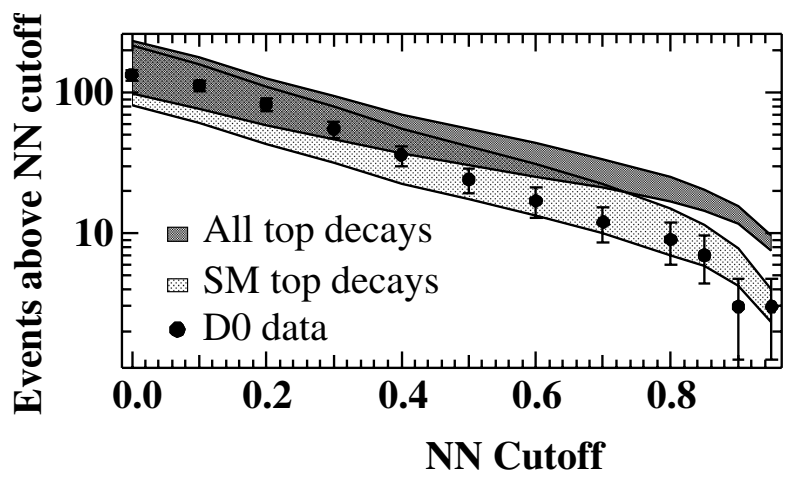

FIG. 2. Data and the number of events expected from all SM backgrounds (light), and from extra $H^{ \pm}$sources (dark) for $\tan \beta=150$ and $M_{H^{ \pm}}=95 \mathrm{GeV}$, as a function of $\mathrm{NN}$ threshold. 
primary sources of systematic uncertainties. The first two are calculated as in Ref. [22], while uncertainty in $\tau$ identification is calculated as in Ref. [17].

Had $H^{ \pm}$bosons been produced in $t \bar{t}$ decays, then the number of $t \bar{t} \rightarrow \tau+$ jets events would have exceeded expectation of the SM at high $\tan \beta$, because $B\left(H^{+} \rightarrow\right.$ $\left.\tau^{+} \nu_{\tau}\right)=0.96$ in this region, while $B\left(W^{+} \rightarrow \tau \nu_{\tau}\right)=$ 0.11 . Consequently, large $\tan \beta$ should be especially sensitive to contributions from $H^{ \pm}$. However, our data agree with the SM. Hence, to set a limit, we calculate the proba- bility for data to fluctuate to the expectation from $H^{ \pm}$ sources. Figure 2 shows the number of events observed, the number expected from SM processes, and the excess from $H^{ \pm}$for $\tan \beta=150$ and $M_{H^{ \pm}}=95 \mathrm{GeV}$, as a function of NN threshold. Above our NN cutoff of 0.91 , there is clear inconsistency with the hypothesis of excess $\tau$ production from $H^{ \pm}$sources.

The probability that the number of expected events for a particular value of $\tan \beta$ and $M_{H^{ \pm}}$has fluctuated to the number of observed events $\left(n_{\text {obs }}\right)$ is given by the joint posterior probability density for $M_{H^{ \pm}}$and $\tan \beta$ :

$$
P\left(M_{H^{ \pm}}, \tan \beta \mid n_{\mathrm{obs}}\right) \propto \int G(\mathcal{L}) \int G\left(n_{B}\right) \int G(A) \times P\left(n_{\mathrm{obs}} \mid \mu\right) d A d n_{B} d \mathcal{L},
$$

where $G$ represents Gaussian distributions, $n_{B}$ is the number of expected background events, and $P\left(n_{\mathrm{obs}} \mid \mu\right)$ is the Poisson probability of $n_{\text {obs }}$ events, given expectation: $\mu\left(M_{H^{ \pm}}, \tan \beta\right)=A\left(M_{H^{ \pm}}, \tan \beta\right) \sigma(t \bar{t}) \mathcal{L}+n_{B}$, where $A\left(M_{H^{ \pm}}, \tan \beta\right)$ is the sum of the products of the branching fractions and efficiencies from all sources of $t \bar{t}$ decay. For a particular $M_{H^{ \pm}}$, and any $\tan \beta$, the value of $A$ is computed via MC (in leading order). The probabilities from Eq. (1) are then parametrized as a function of $\tan \beta$ for fixed values of $M_{H^{ \pm}}$, and fitted as a function of $M_{H^{ \pm}}$ to obtain $P\left(M_{H^{ \pm}}, \tan \beta \mid n_{\text {obs }}\right)$, the Bayesian posterior probability density [24] shown in Fig. 3.

The prior probability distribution, as in the indirect search [4], is assumed to be uniform over the allowed regions of $M_{H^{ \pm}}$and $\log _{10}(\tan \beta)$ and zero elsewhere. This gives equal weight to all possible branching ratios in type II THDM. We further impose a lower limit on $M_{H^{ \pm}}$of $75 \mathrm{GeV}$, to provide an overlap with the limit from LEP experiments. The C.L. exclusion boundary in the $\left(M_{H^{ \pm}}, \tan \beta\right)$ plane is obtained by integrating the probability density $P\left(M_{H^{ \pm}}, \tan \beta \mid n_{\mathrm{obs}}\right)$ around a contour of constant $P$, such that the volume under the surface enclosed by that contour constitutes $95 \%$ of the volume

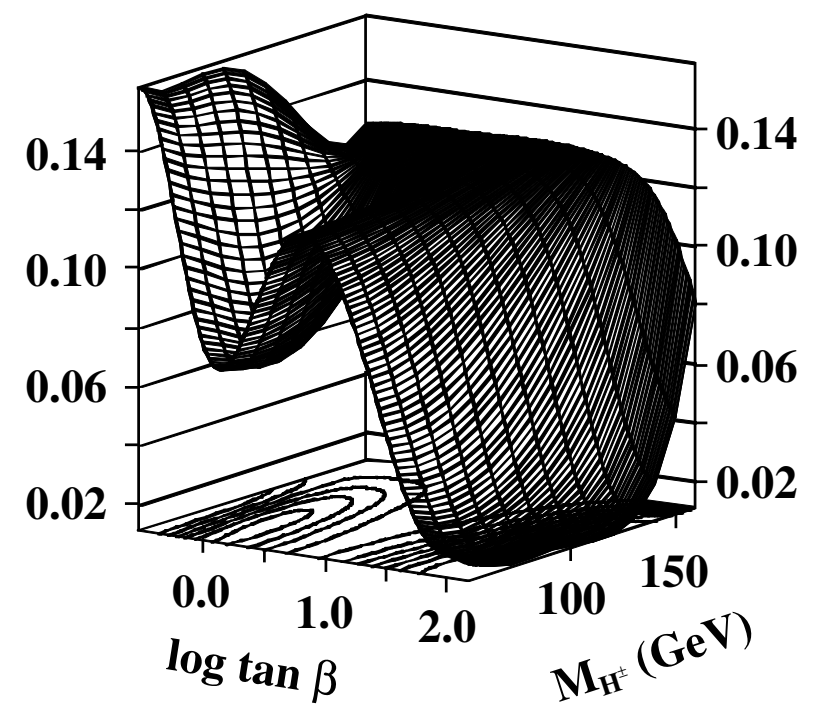

FIG. 3. The normalized surface for $P\left(M_{H^{ \pm}}, \tan \beta \mid n_{\mathrm{obs}}\right)$. under the full $P\left(M_{H^{ \pm}}, \tan \beta \mid n_{\text {obs }}\right)$ surface. A $10 \%$ change in the $t \bar{t}$ cross section changes the excluded region by $10 \%$, with the larger cross section yielding greater exclusion. The limits are shown in Fig. 4, along with results from our indirect D0 search, under the same assumptions. The exclusion region correspond to parameters that are $<5 \%$ likely. Because the indirect search excludes simultaneously both large and small $\tan \beta$, the exclusion contour at high $\tan \beta$ represents approximately $2.5 \%$ of the volume under that posterior probability density surface. Also shown in Fig. 4 are the frequentist limits, wherein a point in the $\left(M_{H^{ \pm}}, \tan \beta\right)$ plane is excluded when $P\left(n_{\text {obs }} \mid M_{H^{ \pm}}, \tan \beta\right)<5 \%$, which is related to the posterior probability through Bayes theorem [24]. Although the frequentist and Bayesian exclusion contours are shown on the same plot, they represent entirely different notions of probability [24].

In summary, our direct search for charged Higgs bosons in top quark decays shows no evidence of signal for $M_{H^{ \pm}}<150 \mathrm{GeV}$. The region of small $\tan \beta$ does not provide $\tau$ leptons through couplings to $H^{ \pm}$, and therefore cannot be excluded. At large $\tan \beta$, we extend the exclusion region beyond that of our indirect search. Assuming $m_{t}=175 \mathrm{GeV}$ and $\sigma(t \bar{t})=5.5 \mathrm{pb}, \tan \beta>32.0$ is excluded at the $95 \%$ C.L., for $M_{H^{ \pm}}=75 \mathrm{GeV}$. The limits are less stringent at larger $M_{H^{ \pm}}$, until $M_{H^{ \pm}}=150 \mathrm{GeV}$,

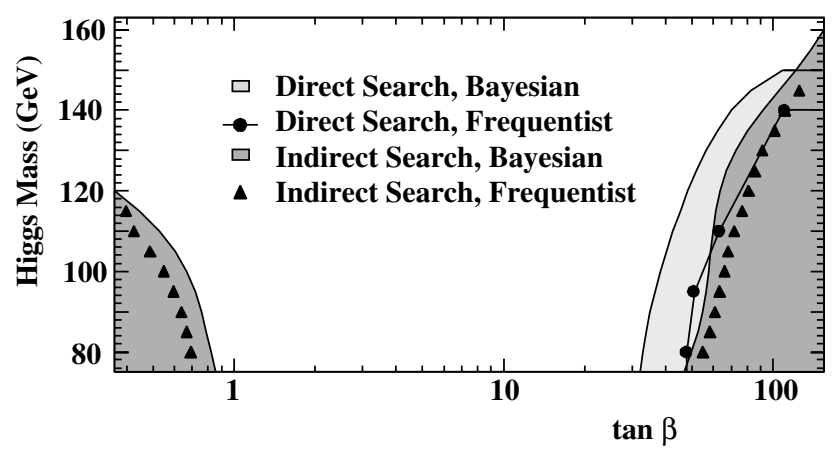

FIG. 4. The region of exclusions at $95 \%$ C.L. in $\left(M_{H^{ \pm}}, \tan \beta\right)$ for $m_{t}=175 \mathrm{GeV}$ and $\sigma(t \bar{t})=5.5 \mathrm{pb}$. (When statistical and systematic uncertainties become large, the Bayesian limit can depend on the distribution assumed for the prior probability.) 
where no limit can be set. Using the results of this Letter and those of our indirect search, we exclude $B(t \rightarrow$ $\left.H^{+} b\right)>0.36$ at $95 \%$ C.L. in the region $0.3<\tan \beta<$ 150 , and $M_{H^{ \pm}}<160 \mathrm{GeV}$.

We thank the staffs at Fermilab and collaborating institutions, and acknowledge support from the Department of Energy and National Science Foundation (USA), Commissariat à L'Energie Atomique and CNRS/Institut National de Physique Nucléaire et de Physique des Particules (France), Ministry for Science and Technology and Ministry for Atomic Energy (Russia), CAPES and CNPq (Brazil), Departments of Atomic Energy and Science and Education (India), Colciencias (Colombia), CONACyT (Mexico), Ministry of Education and KOSEF (Korea), CONICET and UBACyT (Argentina), The Foundation for Fundamental Research on Matter (The Netherlands), PPARC (United Kingdom), Ministry of Education (Czech Republic), and the A.P. Sloan Foundation.

[1] P. W. Higgs, Phys. Lett. 12, 132 (1964); Phys. Rev. Lett. 13, 508 (1964); Phys. Rev. 145, 1156 (1966); F. Englert and R. Brout, Phys. Rev. Lett. 13, 321 (1964); G. S. Guralnik, C. R. Hagen, and T. W. B. Kibble, Phys. Rev. Lett. 13, 585 (1964).

[2] J.F. Gunion, H.E. Haber, G. Kane, and S. Dawson, The Higgs Hunters Guide (Addison-Wesley, Redwood City, California, 1990), p. 200.

[3] E. Ma, D. P. Roy, and J. Wudka, Phys. Rev. Lett. 80, 1162 (1998).

[4] D0 Collaboration, B. Abbott et al., Phys. Rev. Lett. 82, 4975 (1999).

[5] LEP Collaborations, hep-ex/0107031.

[6] CDF Collaboration, F. Abe et al., Phys. Rev. Lett. 79, 357 (1997); CDF Collaboration, T. Affolder et al., Phys. Rev. D 62, 012004 (2000).
[7] CLEO Collaboration, M.S. Alam et al., Phys. Rev. Lett. 74, 2885 (1995).

[8] L3 Collaboration, M. Acciarri et al., Phys. Lett. B 396, 327 (1997).

[9] M. L. Mangano and S. R. Slabospitsky, Phys. Lett. B 410, 299 (1997).

[10] S. Towers, hep-ex/0004022 [Phys. Lett. B (to be published)].

[11] E. Smith, Ph.D. thesis, University of Oklahoma, 1999 (unpublished).

[12] D0 Collaboration, S. Abachi et al., Nucl. Instrum. Methods Phys. Res., Sect. A 338, 185 (1994).

[13] For an example, see B. Muller, J. Reinhardt, and M. T. Strickland, Neural Networks: An Introduction (SpringerVerlag, New York, 1995), p. 13.

[14] C. Peterson and T. Rögnavaldsson, CERN-TH.7135/94, 1994 (unpublished).

[15] F. Paige and S. Protopopescu, BNL Report No. BNL38034, 1986 (unpublished). We used version 7.21.

[16] F. A. Berends, H. Kuijf, B. Tausk, and W. T. Giele, Nucl. Phys. B357, 32 (1991).

[17] D0 Collaboration, B. Abbott et al., Phys. Rev. Lett. 84, 5710 (2000).

[18] D0 Collaboration, B. Abbott et al., Phys. Rev. D 60, 052001 (1999).

[19] E. L. Berger and H. Contopanagos, Phys. Rev. D 54, 3085 (1996).

[20] S. Catani, M. L. Mangano, P. Nason, and L. Trentadue, Phys. Lett. B 378, 329 (1996).

[21] E. Laenen, J. Smith, and W. L. van Neerven, Phys. Lett. B 321, 254 (1994).

[22] D0 Collaboration, D. B. Abbott et al., Phys. Rev. D 58, 052001 (1998).

[23] CDF Collaboration, F. Abe et al., Phys. Rev. Lett. 82, 271 (1999).

[24] E. T. Jaynes, "Probability Theory: The Logic of Science" (to be published). Copies of the manuscript are available from http://bayes.wustl.edu 